\title{
Use of a cardiac occluder for closure of a complex gastric leak after bariatric surgery
}

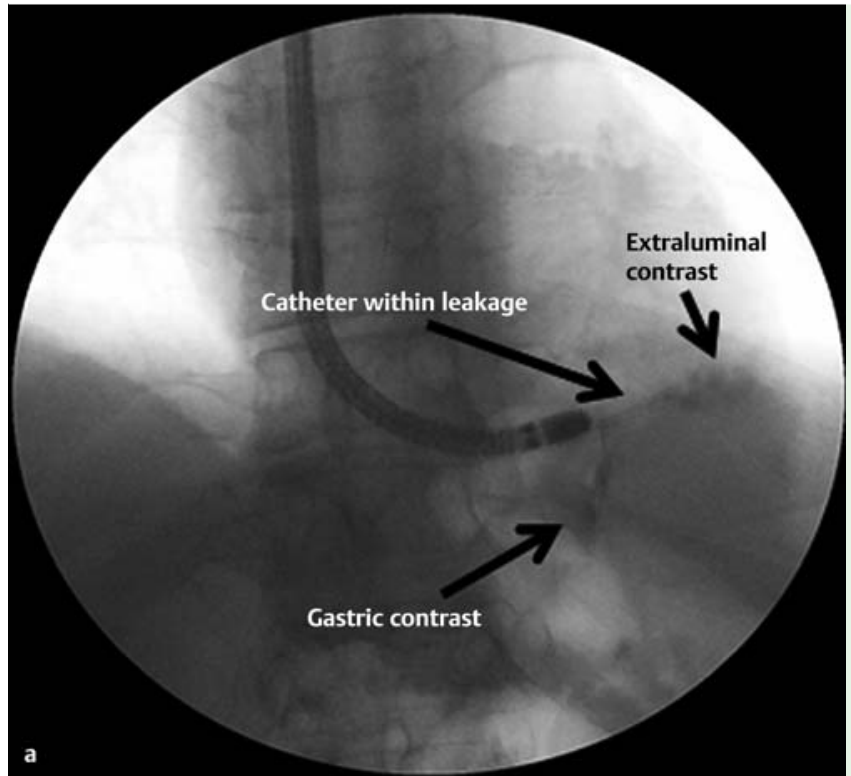

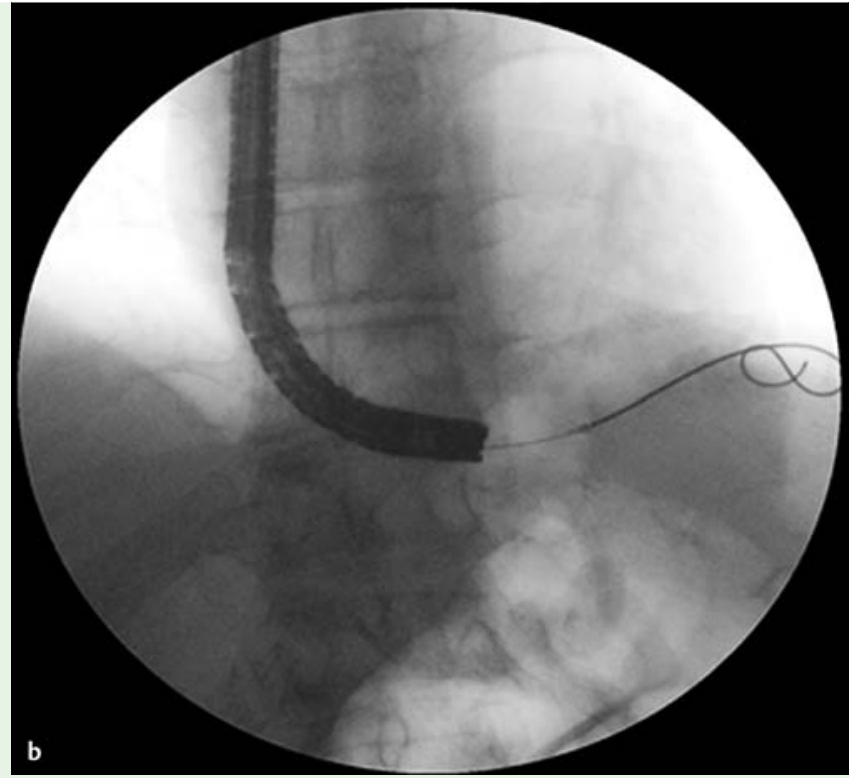

Fig. 1 Radiological images during deployment of the ventricular septal defect (VSD) occluder. a The endoscope tip is seen within the gastric leak, and extraluminal paragastric contrast agent is visible. b Placement of a guidewire through the gastric wall into the paragastric space to guide application of the VSD occluder. c Image after deployment of the occluder, showing luminal and extraluminal discs with neck in between.
Gastric leakage after sleeve gastrectomy is reported in up to $9 \%$ of cases [1]. Endoscopic measures to manage such complications include insertion of self-expandable metal stents, and application of endoclips or over-the-scope clips [1]. Interventional cardiologists use specialist Amplatzer Occluders (St. Jude Medical, Plymouth, Minnesota, United States) for closure of patent foramen ovale, atrial septal defects, or ventricular septal defects (VSDs) [2]. The Amplatzer Occluder is a self-expand- able double-disc device made from a nitinol wire mesh. Dacron polyester patches are sewn into each disc and the connecting waist in order to enhance endocardialization of the device. The device was specifically designed for the muscular VSD, and features a neck that is $5-10 \mathrm{~mm}$ in length, commensurate with the defect, and a slightly larger retention disc on the distal (left) side. This report describes the use of such a device to close a complex gastric leak after bariatric surgery.
A 40-year-old morbidly obese man (body mass index $47.6 \mathrm{~kg} / \mathrm{m}^{2}$, noninsulin-dependent diabetes, arterial hypertension, and obstructive sleep apnea syndrome) underwent gastric sleeve resection in July 2012. A proximal leakage was treated on postoperative Day 7 by endoluminal stent graft application and re-laparoscopic switch to gastric-bypass with Roux Yanastomosis. However, even prolonged stent graft application (including repetitive exchange and endoscopic re-evalua- 


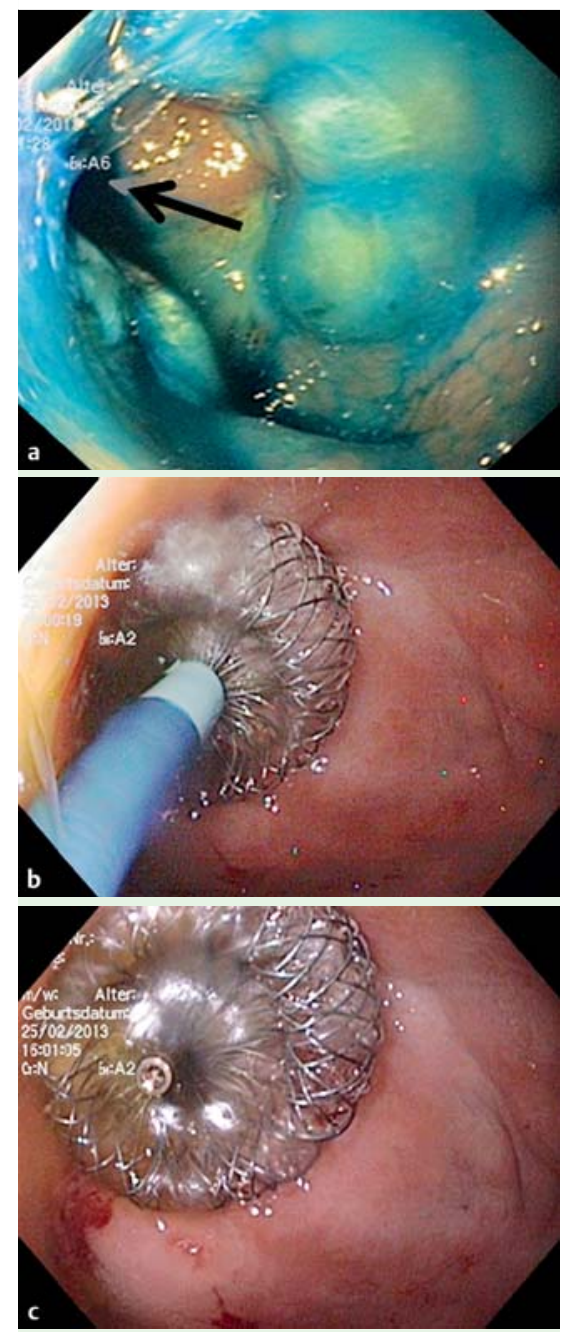

Fig. 2 Endoscopic images during deployment of the ventricular septal defect (VSD) occluder. a Endoscopic view from the gastroesophageal junction. There is leakage in the cardia area (arrow points towards extraluminal space). The blue color is methylene blue, used to visualize and diagnose the leakage into the paragastric space. $\mathbf{b}$ Deployment of the VSD occluder. c The VSD occluder in place, viewed from the stomach. tion) did not lead to closure of the leak. Therefore, 7 months after detection of the proximal gastric leak, an Amplatzer VSD Occluder was placed in a final attempt at endoscopic closure before surgery was indicated.

A neck diameter of $12 \mathrm{~mm}(7 \mathrm{~mm}$ in length), which was about 2-4 mm larger than the size of the leak, was judged to be appropriate. The procedure was performed under general anesthesia. Under endoscopic view of the leak and radiological confirmation by contrast agent ( $\bullet$ Fig. 1), a stiff Back-up Meier guidewire (Boston Scientific Corp., Natick, Massachusetts, United States) was used to guide a TorqVue sheath (St. Jude Medical) across the gastric wall defect. The dilator and the wire were removed, and the VSD occluder was loaded and deployed ( $\bullet$ Fig. 2 ).

The patient later developed a second leak at the gastrojejunostomy, which was treated surgically. However, the initial leak closed completely, and the Amplatzer Occluder became fully integrated into the tissue, as observed at the most recent endoscopic follow-up in February 2014.

\section{Endoscopy_UCTN_Code_TTT_1AO_2AI}

\section{Competing interests: None}

\section{Reiner Wiest ${ }^{1}$, Radu Tutuian', Bernhard Meier ${ }^{2}$, Philipp Nett ${ }^{1}$}

${ }^{1}$ Department of Visceral Surgery and Medicine, Bern University Hospital Inselspital, Bern, Switzerland

${ }^{2}$ Department of Cardiology, Bern University Hospital Inselspital, Bern, Switzerland

\section{References}

1 Jurowich C, Thalheimer A, Seyfried $F$ et al. Gastric leakage after sleeve gastrectomy clinical presentation and therapeutic options. Langenbecks Arch Surg 2011; 396 : 981-987

2 Wahl A, Meier B. Patent foramen ovale and ventricular septal defect closure. Heart 2009; 95: 70-82

\section{Bibliography}

Dol http://dx.doi.org/

10.1055/s-0034-1377591

Endoscopy 2014; 46: E487-E488

(c) Georg Thieme Verlag KG

Stuttgart · New York

ISSN 0013-726X

\section{Corresponding author \\ Reiner Wiest, MD}

Department Visceral Surgery and Medicine Bern University Hospital Inselspital Inselspital

Bern 3010

Switzerland

Fax: +41-31-6325900

reiner.wiest@insel.ch 